\title{
EFICÁCIA DO TRATAMENTO DE AVG NO CONTROLE DA QUEDA E MATURAÇÃO DOS FRUTOS DE MAÇÃ, CULTIVAR IMPERIAL GALA ${ }^{1}$
}

\author{
JOSÉ LUIS PETRI², GABRIEL BERENHAUSER LEITE ${ }^{3}$ LUIS CARLOS ARGENTA³
}

RESUMO - O objetivo deste trabalho foi avaliar o efeito de concentrações e épocas de aplicação do AVG no controle da queda précolheita, no retardamento da maturação e na qualidade de maçãs cv. Imperial Gala. Testaram-se 4 épocas de aplicação (45; $30 ; 15$ e 7 Dias Antes do início do Ponto de Colheita presumido da testemunha - DAPC) e 4 concentrações $\left(0 ; 90 ; 124\right.$ e $62+62$ g.ha $\left.{ }^{-1}\right)$, em dois anos, 2003 e 2004. A degradação do amido, a queda da firmeza da polpa, o aumento do teor de sólidos solúveis totais (SST) e as mudanças da cor dos frutos tratados com AVG foram retardados em relação aos frutos não-tratados. Conforme esperado, AVG retardou o ponto de colheita comercial em 7 a 16 dias e a queda pré-colheita dos frutos em até 30 dias. Os efeitos do AVG no retardamento da maturação foram dependentes da concentração e da época de aplicação. Aplicações de AVG mais próximas do ponto de colheita comercial, 7 DAPC, foram mais efetivas no controle de queda de frutos pré-colheita e no retardamento da perda de firmeza do que aplicações mais precoces (30 ou 45 DAPC). Doses de 124 e 90 g.ha-1 de AVG são igualmente efetivas quando aplicadas 7 ou 15 dias antes do ponto de colheita comercial. O retardamento da maturação e o controle da queda pré-colheita foi tanto maior quanto maior a dose e mais próximo do ponto de colheita comercial foi a aplicação do AVG. Os resultados demonstram que é possível manejar a intensidade de retardamento da colheita alterando a época de aplicação entre 7 e 30 DAPC e a dose de AVG entre 90 a 124 g.ha ${ }^{-1}$. Termos para indexação: Malus domestica Borkh, biorreguladores, maturação, manejo da colheita, aminothoxyvinilglicine.

\section{EFFICIENCY OF THE TREATMENT OF 'AVG' ON THE FALL AND MATURATION CONTROL OF APPLE FRUIT, 'IMPERIAL GALAI’ CULTIVAR}

\begin{abstract}
The objective of this study was to evaluate the effects of rates and time of AVG (aminoethoxyvinylglycine) application on pre-harvest fruit drop control, ripening delay, and quality of Imperial Gala apple fruit. It was tested 4 application times (45, 30, 15 and 7 days before the predicted harvest date for the control plot - DBPH), and 4 AVG rates $\left(0,90,124\right.$, and $62+62$ g.ha $\left.{ }^{-1}\right)$, during the growing seasons 2003 and 2004. The starch degradation, the loss of firmness, the increase of soluble solids content (SSC), and the development of fruit color were slower on AVG treated than on untreated fruit. As expected, AVG delayed the commercial harvest date by 7 to 16 days, and the pre-harvest fruit drop up to 30 days. The ripening delay by AVG depended on rate and time of application. AVG applied closer to the predicted commercial harvest time (7 DBPH), was more effective on preventing pre-harvest fruit drop and on delaying fruit softening than earlier applications (30 or $45 \mathrm{DBPH})$. AVG applied at the rates of 90 and 124 g.ha-1 were equally effective when applied at 7 or $15 \mathrm{DBPH}$. The delay on fruit ripening and the control of pre-harvest fruit drop by AVG were greater at its highest rates and as closer the application was to the predicted harvest date for the control. The results showed that it is possible to manage de degree of harvest delay by varying the time of application from 7 to $30 \mathrm{DBPH}$, and the rates of AVG from 90 to 124 g.ha-1 .
\end{abstract}

Index terms: Malus domestica Borkh, Aminothoxyvinilglicine

\section{INTRODUÇÃO}

A “Gala", e seus clones, é a cultivar de macieira mais plantada no Brasil, respondendo por mais de $50 \%$ da produção estimada em 980 mil toneladas no ciclo 2004-2005. Essa cultivar caracteriza-se por uma maturação rápida no período de colheita comercial (Argenta, 1992) e pela sensibilidade à queda précolheita dos frutos. Como grande parte dos pomares de macieira no Brasil tem área superior a 50 ha, parte dos frutos é colhida duas a três semanas após o ponto de colheita, em estágio avançado de maturação, ocorrendo uma porcentagem de queda de frutos considerável. Para evitar a queda de frutos, até duas aplicações de ANA (Ácido Naftaleno Acético) se fazem necessárias, acelerando deste modo a perda de resistência da polpa. Duas aplicações de ANA ou a ocorrência de temperaturas elevadas após a primeira aplicação aceleram a redução da firmeza dos frutos (Smock e Gross, 1947).

Aminoethoxyvinylglicine (AVG), inibidor da síntese de etileno, aparece como uma alternativa ao uso do ANA. A aplicação antes do ponto de colheita previne a queda pré-colheita e retarda a maturação dos frutos da macieira (Byers, 1997; Greene, 2002), mas os resultados podem variar de acordo com a cultivar e a concentração utilizada (Bramlage et al., 1980; Autio e Bramlage, 1982; Greene, 2003). Segundo Chun et al. (1997), a época de aplicação influenciou mais do que a concentração, na cv. Tsugaro. Greene (2002) salienta que é possível reduzir a concentração do produto aplicado e controlar a queda de frutos, através de aplicações próximas do ponto de colheita. Segundo Schupp e Greene (2004), a duração do controle da queda de frutos aumentou linearmente com o aumento da concentração

1 (Trabalho 141-06). Recebido em : 13-09-2006. Aceito para publicação em : 16-03-2007.

${ }^{2}$ Eng. Agr., M.Sc., Epagri - Estação Experimental de Caçador, C.P. 591, 89500-000, Caçador- SC. E-mail: petri@epagri.rct-sc.br

${ }^{3}$ Eng. Agr., Dr., Epagri - Estação Experimental de Caçador, C.P. 591, 89500-000, Caçador- SC. E-mail: gabriel@epagri.rct-sc.br, argenta@epagri.rctsc.br 
de AVG, principalmente nas aplicações tardias, mais próximas do ponto de colheita. O uso de AVG retardou a colheita na cv. McIntosh de 7 a 10 dias sem aumentar a queda de frutos (Stover et al., 2003). O retardamento da maturação aumenta o ciclo de floração-maturação, podendo aumentar o tamanho dos frutos. Segundo Greene (1996), os frutos aumentam seu tamanho em 1\% a cada dia a mais que permaneceram na planta.

Os efeitos do AVG sobre firmeza da polpa, sólidos solúveis totais, acidez e degradação de amido podem variar em relação à cultivar. Chun et al. (1997) observaram, na cv. McIntosh, que o AVG retardou a degradação de amido e a perda de firmeza, enquanto Autio e Bramlage (1982) não observaram a mesma resposta na cv. Puritan. Greene (2000), trabalhando com a cv. Ace Delicious, não observou, efeito do AVG sobre a firmeza da polpa, mas observou redução de firmeza na última data avaliada. Segundo Lurie (2000), AVG reduz o desenvolvimento da cor vermelha dos frutos. Trabalhos desenvolvidos no Brasil também mostraram retardamento da maturação de maçãs Gala pelo AVG (Amarante, 2002). No entanto, os fatores ambientais que podem afetar a eficácia do AVG na cv. Gala, não foram ainda claramente definidos.

O objetivo deste trabalho foi avaliar o efeito de concentrações e épocas de aplicação do AVG no controle da queda pré-colheita, no retardamento da maturação e na qualidade dos frutos da macieira cv. Imperial Gala.

\section{MATERIAL E MÉTODOS}

Os experimentos foram conduzidos em pomares comerciais em Fraiburgo, Sul do Brasil (Lat 26 $46^{\prime} \mathrm{S}$, Long $51^{\circ}$ W), nos ciclos 2002-2003 e 2003-2004.

Em 2002-2003, utilizou-se "Imperial Gala"/M-106, com 4 épocas de aplicação, 45; 30; 15 e 7 Dias Antes do início do Ponto de Colheita (DAPC) com base na testemunha, e 3 concentrações, 0; 90 e 124 g.ha $^{-1}$ de AVG $\left(\right.$ Retain $^{\mathrm{TM}}$ ). O delineamento experimental foi em blocos ao acaso, com 5 repetições de 2 plantas por parcela.

Em 2003-2004, utilizou-se "Imperial Gala" /Marubakaido com interenxerto de M-9, com 3 épocas de aplicação $(30 ; 15$ e 7 DAPC), 4 concentrações (0; 90; 124 e 124 g.ha- ${ }^{-1}$ dividido em duas épocas - 62 g.ha-1 a 30 DAPC e 62 g.ha-1 15 DAPC). O delineamento experimental foi um fatorial $3 \times 4$, em blocos casualisados, com 5 repetições de 2 plantas por parcela.

Nos dois ciclos, os tratamentos foram aplicados entre $9 \mathrm{e}$ 10 horas da manhã, utilizando-se de um pulverizador costal motorizado, com bico leque D-S, e um volume de calda de $1.000 \mathrm{~L}$ por ha. Em todos os tratamentos, foi adicionado espalhante siliconado a $0,05 \%$.

A partir do início da maturação dos frutos do tratamentotestemunha ( 0 g.ha ${ }^{-1}$ de AVG), em 5 plantas por tratamento, coletaram-se, semanalmente, frutos para análise de firmeza da polpa, sólidos solúveis totais (SST), índice iodo-amido, acidez titulável, porcentagem de coloração vermelha da superfície dos frutos, cor de fundo, rachadura peduncular e porcentagem de queda de frutos. Nas outras 5 plantas, onde não foram coletadas amostras de frutos, avaliaram-se a porcentagem de frutos colhidos nas diferentes datas de colheita, o peso médio dos frutos, a produção por planta e a porcentagem de coloração vermelha da superfície dos frutos. A porcentagem de frutos colhidos foi determinada pela análise visual da cor de fundo dos frutos. Todos os frutos considerados maduros, em cada planta, foram colhidos e contados semanalmente. A porcentagem de frutos colhidos foi determinada pela relação do número de frutos colhidos e o número total de frutos de cada planta, previamente contados. Os índices de maturação (firmeza da polpa, índice de iodo-amido e acidez titulável) foram determinados semanalmente, numa amostra de 5 frutos por planta, colhidos na parte mediana da planta. As análises foram realizadas conforme descritos por Argenta (1992).

\section{RESULTADOS E DISCUSSÃO}

Maturação dos frutos: Aplicação de AVG retardou a maturação dos frutos na planta. A queda da firmeza da polpa, a degradação do amido, o aumento do teor de sólidos solúveis totais (SST) e as mudanças da cor dos frutos tratados com AVG foram retardados em relação aos frutos não-tratados (Tabela 3; 4; 5; 6 e 7). Em 2002-2003, a firmeza dos frutos tratados com AVG 90 g.ha $^{-1} 15$ DAPC foi superior àquela dos demais tratamentos com AVG na última data de avaliação, em 25-03 (Tabela 4). Em 2003-2004, a firmeza da polpa nos tratamentos de 15 e 7 DAPC manteve-se significativamente superior, até o dia 17-03, em relação ao tratamento-testemunha e ao tratamento 30 DAPC (Tabela 3).

Stover et al. (2003) observaram que o efeito do AVG sobre a queda de frutos e a firmeza de polpa varia entre local e ano de aplicação. As razões para essas diferenças ainda não são totalmente conhecidas, mas a evolução mais lenta da síntese de etileno em condições de temperaturas mais amenas, na época de maturação dos frutos, poderia induzir a um efeito mais prolongado do AVG sobre o etileno, pois baixas temperaturas normalmente aumentam a capacidade de produção de etileno. Portanto as condições climáticas são importantes.

Não houve diferença significativa quanto ao índice de amido e cor de fundo entre os tratamentos com AVG, mas todos diferiram significativamente da testemunha. Apesar de ter sido retardada, a coloração vermelha dos frutos atingiu os mesmos níveis ou levemente inferiores comparados à testemunha, quando colhidos no ponto de colheita comercial (Tabela 7).

O controle da maturação pelo AVG resultou em retardamento da colheita (Tabelas 1 e 2). A percentagem de frutos colhidos (no ponto de maturação comercial) foi alta para frutostestemunha nas primeiras datas de colheita, e altas para frutos tratados com AVG nas últimas datas de colheita. Em 2002-2003, nos tratamentos com AVG, o início da colheita foi retardado de 7 a 11 dias em relação ao tratament-testemunha (sem AVG), independentemente da época e da concentração utilizada (Tabela 1). Em 2003-2004, o final da colheita foi retardado em 16 dias. A porcentagem de frutos colhidos, em cada data de colheita, foi influenciada pela época e concentração de AVG. Aplicações de 7 e 15 DAPC apresentaram menor percentual de frutos colhidos 
até 2-03 (data de encerramento da colheita da testemunha) em relação ao tratamento de AVG $120 \mathrm{~g} /$ ha aplicado 30 DAPC (Tabela $2)$. Até 23-02, a porcentagem de frutos colhidos era de aproximadamente $67 \%$, no tratamento-testemunha, e de 3 a $9 \%$ nos tratamentos com AVG (Tabela 2). Esses resultados estão de acordo com Stover et al. (2003), que, utilizando uma concentração de 124 g.ha- ${ }^{-1}$, observaram um retardamento de 7 a 10 dias na colheita da cv. McIntosh. Aplicação de AVG 45 DAPC aumentou o percentual de frutos colhidos em 25-02 e 05-03 em relação às aplicações 15 e 7 DAPC (Tabela1). Isto demonstra que tratamentos 15 e 7 DAPC retardam mais a colheita dos frutos. Aplicação de AVG $62 \mathrm{~g} / \mathrm{ha}$, em duas vezes, aos 15 e 7 DAPC, não mostrou diferença significativa em relação a uma aplicação de 124 g.ha-1 .

Com o retardamento da colheita, os frutos continuaram crescendo, refletindo-se no aumento do peso médio dos mesmos, nas diferentes datas de colheita e na média geral, em ambos os anos estudados. Porém não houve diferença significativa entre época de aplicação e concentração (Tabelas 8 e 9). No ciclo 20032004 o aumento do peso médio dos frutos em relação ao tratamento-testemunha variou de $9,1 \%$ a $16,3 \%$ nos tratamentos de AVG. Segundo Greene (2004), o atraso de 2 a 3 semanas na colheita resulta em aumento de $15 \%$ a $20 \%$ no peso dos frutos, resultando num aumento de uma a duas classes na classificação dos frutos, refletindo, deste modo, em preços melhores de venda.

\section{Queda de frutos na pré-colheita}

AVG reduziu significativamente a queda de frutos na précolheita, mantendo os níveis de queda inferiores ao tratamentotestemunha por um período de mais de 30 dias após o início da colheita (Tabelas 10 e 11).

Aplicações mais próximas do ponto de colheita foram mais efetivas que aplicações 30 e 45 DAPC (Tabela 10), sendo que a maior eficiência foi obtida no tratamento 15 DAPC, para as duas concentrações estudadas.

Em 2003-2004, até 10-03, cerca de 26 dias após o PC do tratamento-testemunha, todos os tratamentos de AVG diferiram significativamente da testemunha, porém não houve diferenças entre época e concentração de AVG (Tabela 11). Em 25-03, o tratamento de 124 g.ha- ${ }^{-1}$ de AVG aplicado aos 7 DAPC foi o que apresentou o menor percentual de queda de frutos. Isto demonstra que a eficiência no controle da queda de frutos diminui ao longo do tempo após a aplicação de AVG, período este que poderá variar com a concentração e condições ambientais. Os resultados indicam que o controle da queda de frutos permanece a níveis toleráveis comercialmente por 15 a 30 dias após a colheita do tratamento-testemunha, variando principalmente com a época de aplicação.

Os resultados indicam que é possível manejar a intensidade de retardamento da colheita, selecionando a época de aplicação entre 7 e 30 DAPC e a dose de AVG entre 90 e 124 g.ha-1 ${ }^{-1}$

O retardamento da maturação e o controle da queda précolheita é tanto maior quanto maior a dose, se aplicado próximo do ponto de colheita comercial.
TABELA 1 - Efeito da época de aplicação de AVG, independentemente da concentração utilizada, na porcentagem acumulada de frutos colhidos na cv. Imperial Gala, em diversas datas de avaliações. Fraiburgo-SC, 2003.

\begin{tabular}{lcccc}
\hline \multirow{2}{*}{ Época de Aplicação } & \multicolumn{4}{c}{$\%$ de frutos colhidos - dados acumulados } \\
\cline { 2 - 5 } & $\mathbf{1 8 - 0 2}$ & $\mathbf{2 5 - 0 2}$ & $\mathbf{0 5 - 0 3}$ & $\mathbf{1 7 - 0 3}$ \\
\hline Sem aplicação & 50,5 & 65,5 & 87,6 & 100 \\
45 DAPC* & 0,0 & 39,3 & 71,0 & 100 \\
30 DAPC & 0,0 & 40,5 & 74,9 & 100 \\
15 DAPC & 0,0 & 18,6 & 52,3 & 100 \\
7 DAPC & 0,0 & 15,7 & 38,3 & 100 \\
\hline
\end{tabular}

*Dias Antes do Ponto de Colheita

TABELA 2 - Efeito de concentrações e épocas de aplicação de AVG, na porcentagem acumulada de frutos colhidos na cv. Imperial Gala, em diversas datas de avaliações. Fraiburgo- SC, 2004.

\begin{tabular}{|c|c|c|c|c|c|c|c|}
\hline \multirow{2}{*}{\multicolumn{2}{|c|}{$\begin{array}{c}\text { Tratamentos } \\
\text { AVG }\end{array}$}} & \multicolumn{6}{|c|}{$\%$ de frutos colhidos - acumulado } \\
\hline & & 12-02 & 19-02 & 23-02 & $02-03$ & $10-03$ & 18-03 \\
\hline $0 \mathrm{~g} \cdot \mathrm{ha}^{-1}$ & & 5,8 & 23,4 & $67,7 \mathrm{a}$ & $100,0 \mathrm{a}$ & 100,0 n.s. & 100,0 \\
\hline 124g.ha ${ }^{-1}$ & 30 DAPC* & 0,0 & 0,0 & $9,2 \mathrm{~b}$ & $45,8 \mathrm{~b}$ & 87,3 & 100,0 \\
\hline $\begin{array}{l}\text { 62g.ha }{ }^{-1} \\
\text { 62g.ha }{ }^{-1}\end{array}$ & $\begin{array}{l}30 \text { DAPC + } \\
15 \text { DAPC }\end{array}$ & 0,0 & 0,0 & $3,4 \mathrm{~b}$ & $33,5 \mathrm{bc}$ & 76,4 & 100,0 \\
\hline 90g.ha ${ }^{-1}$ & 15 DAPC & 0,0 & 0,0 & $5,1 \mathrm{~b}$ & $20,3 \mathrm{c}$ & 66,4 & 100,0 \\
\hline 124g.ha ${ }^{-1}$ & 7 DAPC & 0,0 & 0,0 & $7,2 \mathrm{~b}$ & $32,9 \mathrm{bc}$ & 84,1 & 100,0 \\
\hline 90g.ha ${ }^{-1}$ & 7 DAPC & 0,0 & 0,0 & $4,1 b$ & $33,6 \mathrm{bc}$ & 80,6 & 100,0 \\
\hline
\end{tabular}

* Dias Antes do Ponto de Colheita

Médias seguidas da mesma letra, na coluna, não diferiram significativamente, a $5 \%$ de probabilidade, pelo teste de Duncan.

TABELA 3 - Efeito de concentrações e épocas de aplicação de AVG, na firmeza da polpa $\left(\mathrm{lb} / \mathrm{cm}^{2}\right)$, na cv. Imperial Gala,em diversas datas de avaliações. FraiburgoSC, 2004.

\begin{tabular}{|c|c|c|c|c|c|c|c|c|}
\hline \multirow{2}{*}{\multicolumn{2}{|c|}{$\begin{array}{c}\text { Tratamentos } \\
\text { AVG }\end{array}$}} & \multicolumn{7}{|c|}{ Firmeza da polpa $\left(\mathrm{lb} / \mathrm{cm}^{2}\right)$} \\
\hline & & $12-02$ & 19-02 & $26-02$ & $04-03$ & $10-03$ & 17-03 & $26-03$ \\
\hline 0g.ha ${ }^{-1}$ & & $19,0 \mathrm{~b}$ & $17,9 \mathrm{c}$ & $16,7 \mathrm{c}$ & $15,2 \mathrm{c}$ & 14,9 n.s. & $14,5 \mathrm{c}$ & $13, \ln . \mathrm{s}$ \\
\hline 124g.ha & & $19,5 \mathrm{~b}$ & $18,8 \mathrm{bc}$ & $17,5 \mathrm{bc}$ & $16,5 \mathrm{~b}$ & 15,9 & $14,7 \mathrm{c}$ & 13,9 \\
\hline $\begin{array}{l}62 \mathrm{~g} \cdot \mathrm{ha}^{-1} \\
62 \mathrm{~g} \cdot \mathrm{ha}^{-1}\end{array}$ & $\begin{array}{l}30 \text { DAPC + } \\
15 \text { DAPC }\end{array}$ & $19,8 \mathrm{~b}$ & $19,5 \mathrm{ab}$ & $18,3 \mathrm{ab}$ & $17,0 \mathrm{~b}$ & 16,1 & $16,0 \mathrm{ab}$ & 14,3 \\
\hline $90 \mathrm{~g} \cdot \mathrm{ha}^{-1}$ & 15 DAPC & $21,5 \mathrm{a}$ & $20,7 \mathrm{a}$ & $19,1 \mathrm{a}$ & $18,7 \mathrm{a}$ & 17,6 & $16,8 \mathrm{a}$ & 15,1 \\
\hline 124g.ha ${ }^{-1}$ & 7 DAPC & $20,3 \mathrm{ab}$ & $19,7 \mathrm{ab}$ & $18,2 \mathrm{ab}$ & $17,4 \mathrm{~b}$ & 16,9 & $16,6 \mathrm{a}$ & 15,3 \\
\hline 90g.ha ${ }^{-1}$ & 7 DAPC & $20,0 \mathrm{~b}$ & $19,1 \mathrm{bc}$ & $17,9 \mathrm{abc}$ & $16,8 \mathrm{~b}$ & 16,0 & $15,3 \mathrm{bc}$ & 14,3 \\
\hline
\end{tabular}

*Dias Antes do Ponto de Colheita

Médias seguidas da mesma letra, na coluna, não diferiram significativamente, a $5 \%$ de probabilidade, pelo teste de Duncan. 
TABELA 4 - Efeito de concentrações e épocas de aplicação de AVG, na firmeza da polpa da cv. Imperial Gala. Fraiburgo-SC, 2003.

\begin{tabular}{|c|c|c|c|c|c|}
\hline \multirow{2}{*}{\multicolumn{2}{|c|}{$\begin{array}{c}\text { Tratamentos } \\
\text { AVG }\end{array}$}} & \multicolumn{4}{|c|}{ Firmeza da polpa $-\mathrm{lb} / \mathrm{cm}^{2}$} \\
\hline & & 25-02 & 06-03 & $17-03$ & 25-03 \\
\hline 90 g.ha $^{-1}$ & 45 DAPC* & $16,1 \mathrm{c}$ & 14,0 de & 13,7 cde & $11,7 \mathrm{~cd}$ \\
\hline 124 g.ha $^{-1}$ & 45 DAPC & $16,2 \mathrm{bc}$ & 14,0 de & $13,7 \mathrm{abcd}$ & $13,2 \mathrm{~b}$ \\
\hline 90 g.ha $^{-1}$ & 30 DAPC & $17,1 \mathrm{a}$ & $16,1 \mathrm{a}$ & $14,3 \mathrm{ab}$ & $12,9 \mathrm{~b}$ \\
\hline 124 g.ha $^{-1}$ & 30 DAPC & $16,2 \mathrm{bc}$ & $14,8 \mathrm{~cd}$ & $13,8 \mathrm{abc}$ & $13,4 \mathrm{~b}$ \\
\hline 90 g.ha $^{-1}$ & 15 DAPC & $16,8 \mathrm{abc}$ & $14,9 \mathrm{bcd}$ & 13,2 bcde & $13,3 \mathrm{~b}$ \\
\hline 124 g.ha $^{-1}$ & 15 DAPC & $17,0 \mathrm{ab}$ & $15,6 \mathrm{abc}$ & $14,8 \mathrm{a}$ & $14,5 \mathrm{a}$ \\
\hline 0 g.ha ${ }^{-1}$ & 45 DAPC & $15,3 \mathrm{~d}$ & 14,0 de & $12,1 \mathrm{ef}$ & $11,3 \mathrm{~d}$ \\
\hline 0 g.ha $^{-1}$ & 30 DAPC & $14,5 \mathrm{~d}$ & $12,8 \mathrm{f}$ & $11,5 \mathrm{f}$ & $11,8 \mathrm{~cd}$ \\
\hline 0 .ha ${ }^{-1}$ & 15 DAPC & $14,8 \mathrm{~d}$ & 13,2 ef & $12,5 \mathrm{def}$ & $12,5 \mathrm{bc}$ \\
\hline 90 g.ha $^{-1}$ & 7 DAPC & $16,2 \mathrm{bc}$ & $15,8 \mathrm{ab}$ & $14,4 \mathrm{ab}$ & $13,3 \mathrm{~b}$ \\
\hline 124 g.ha $^{-1}$ & 7 DAPC & $16,7 a b c$ & $15,6 a b c$ & $13,9 \mathrm{abc}$ & $13,0 \mathrm{~b}$ \\
\hline
\end{tabular}

*Dias Antes do Ponto de Colheita

Médias seguidas da mesma letra, na coluna, não diferiram significativamente, a $5 \%$ de probabilidade, pelo teste de Duncan.

TABELA 5 - Efeito de concentrações e épocas de aplicação de AVG, no índice iodo amido (0 a 9) na cv Gala, em diversas datas de avaliações. Fraiburgo-SC, 2004.

\begin{tabular}{|c|c|c|c|c|c|c|c|}
\hline \multirow{2}{*}{$\begin{array}{c}\text { Tratamentos } \\
\text { AVG }\end{array}$} & \multicolumn{7}{|c|}{ Índice de iodo amido } \\
\hline & $12-02$ & 19-02 & 26-02 & 04-03 & $10-03$ & $17-03$ & 26-03 \\
\hline 0g.ha ${ }^{-1}$ & $2,5 \mathrm{a}$ & $4,5 \mathrm{a}$ & $6,6 \mathrm{a}$ & $7,1 \mathrm{a}$ & $8,7 \mathrm{a}$ & $8,7 \mathrm{a}$ & $8,9 \mathrm{a}$ \\
\hline 124g.ha ${ }^{-1} 30$ DAPC * & $1,3 \mathrm{ab}$ & $2,6 \mathrm{~b}$ & $4,4 \mathrm{~b}$ & $6,0 \mathrm{ab}$ & $7,5 \mathrm{ab}$ & $8,2 \mathrm{ab}$ & $8,7 \mathrm{ab}$ \\
\hline $\begin{array}{ll}\text { 62g.ha } & 30 \text { DAPC + } \\
\text { 62g.ha } & 15 \text { DAPC }\end{array}$ & $1,1 \mathrm{~b}$ & $1,2 \mathrm{~b}$ & $2,6 \mathrm{~cd}$ & $5,0 \mathrm{bc}$ & $6,5 \mathrm{~b}$ & $7,4 a b c$ & $8,4 \mathrm{ab}$ \\
\hline 15 DAPC & $1,0 \mathrm{~b}$ & $1,0 \mathrm{~b}$ & $2,8 \mathrm{~cd}$ & $4,0 \mathrm{c}$ & $5,7 \mathrm{~b}$ & $7,0 \mathrm{bc}$ & $8,2 \mathrm{~b}$ \\
\hline 124g.ha" 7 DAPC & $1,1 \mathrm{~b}$ & $1,3 \mathrm{~b}$ & $2,3 \mathrm{~d}$ & $4,3 \mathrm{bc}$ & $5,8 \mathrm{~b}$ & $6,8 \mathrm{c}$ & $7,5 \mathrm{c}$ \\
\hline 90g.ha' ${ }^{-1}$ & $1,5 \mathrm{~b}$ & $1,2 \mathrm{~b}$ & $3,7 \mathrm{bc}$ & $5,2 \mathrm{bc}$ & $6,8 \mathrm{ab}$ & $8,2 \mathrm{ab}$ & $8,3 \mathrm{ab}$ \\
\hline
\end{tabular}

*Dias Antes do Ponto de Colheita Médias seguidas da mesma letra, na coluna, não diferiram significativamente, a $5 \%$ de probabilidade, pelo teste de Duncan.

TABELA 6 - Efeito de concentrações e épocas de aplicação de AVG, na porcentagem de sólidos solúveis totais (SST), na cv. Imperial Gala, em diversas datas de avaliações. Fraiburgo-SC, 2004.

\begin{tabular}{|c|c|c|c|c|c|c|c|c|}
\hline \multirow{2}{*}{\multicolumn{2}{|c|}{$\begin{array}{c}\text { Tratamentos } \\
\text { AVG }\end{array}$}} & \multicolumn{7}{|c|}{ SST \% } \\
\hline & & $12-02$ & 19-02 & $26-02$ & $04-03$ & $10-03$ & $17-03$ & 26-03 \\
\hline 0 g.ha $^{-1}$ & & $10,5 \mathrm{a}$ & $11,5 \mathrm{a}$ & $12,0 \mathrm{a}$ & 12,5 n.s. & $13,2 \mathrm{a}$ & 13,6 & 14,1 \\
\hline 124g.ha ${ }^{-1}$ & 30 DAPC $^{*}$ & $9,9 \mathrm{~b}$ & $11,2 \mathrm{ab}$ & $11,6 \mathrm{~b}$ & 12,1 & $12,7 \mathrm{ab}$ & 13,3 & 13,5 \\
\hline $\begin{array}{l}\text { 62g.ha-1 } \\
\text { 62g.ha-1 }\end{array}$ & $\begin{array}{l}30 \text { DAPC + } \\
15 \text { DAPC }\end{array}$ & $10,0 \mathrm{~b}$ & $10,6 \mathrm{~cd}$ & $11,4 b c$ & 12,0 & $12,2 \mathrm{~b}$ & 13,2 & 13,8 \\
\hline 90g.ha ${ }^{-1}$ & 15 DAPC & $10,0 \mathrm{~b}$ & $10,7 \mathrm{~cd}$ & $11,3 \mathrm{bc}$ & 12,0 & $12,8 \mathrm{ab}$ & 13,8 & 14,0 \\
\hline 124g.ha ${ }^{-1}$ & 7 DAPC & $9,9 \mathrm{~b}$ & $11,0 \mathrm{bc}$ & $11,0 \mathrm{c}$ & 12,1 & $12,7 \mathrm{ab}$ & 13,2 & 13,6 \\
\hline $90 \mathrm{~g} \cdot \mathrm{ha}^{-1}$ & 7 DAPC & $9,9 \mathrm{~b}$ & $10,5 \mathrm{~d}$ & $11,3 \mathrm{bc}$ & 11,9 & $12,6 a b$ & 13,2 & 13,7 \\
\hline
\end{tabular}

*Dias Antes do Ponto de Colheita

Médias seguidas da mesma letra, na coluna, não diferiram significativamente, a $5 \%$ de probabilidade, pelo teste de Duncan.
TABELA 7 - Efeito de concentrações e épocas de aplicação de AVG, na cor de fundo dos frutos (graus de 0 a 5 ), na cv. Imperial Gala em diversas datas de avaliações . Fraiburgo- SC, 2004.

\begin{tabular}{|c|c|c|c|c|c|c|c|c|}
\hline \multirow{2}{*}{\multicolumn{2}{|c|}{$\begin{array}{c}\text { Tratamentos } \\
\text { AVG }\end{array}$}} & \multicolumn{7}{|c|}{ Cor de fundo } \\
\hline & & $12-02$ & $19-02$ & $26-02$ & 04-03 & $10-03$ & $17-03$ & $26-03$ \\
\hline 0 g.ha ${ }^{-1}$ & & $2,2 a$ & $2,3 \mathrm{a}$ & $3,1 \mathrm{a}$ & $4,3 \mathrm{a}$ & $4,9 a$ & 4,5 & 4,9 \\
\hline 124g.ha- & $30 \mathrm{DAPC}^{*}$ & $1,1 \mathrm{~b}$ & $2,0 \mathrm{~b}$ & $2,3 \mathrm{~b}$ & $3,2 \mathrm{~b}$ & $4,1 \mathrm{~b}$ & 4,4 & 4,7 \\
\hline $\begin{array}{l}62 \mathrm{~g} \cdot \mathrm{ha}^{-1} \\
62 \mathrm{~g} \cdot \mathrm{ha}^{-1}\end{array}$ & $\begin{array}{l}30 \mathrm{DAPC}+ \\
15 \mathrm{DAPC}\end{array}$ & $1,1 \mathrm{~b}$ & $1,5 \mathrm{c}$ & $2,4 \mathrm{~b}$ & $3,0 \mathrm{~b}$ & $4,0 \mathrm{~b}$ & 4,2 & 4,6 \\
\hline 90g.ha ${ }^{-1}$ & 15 DAPC & $1,1 b$ & $1,5 \mathrm{c}$ & $2,3 \mathrm{~b}$ & $2,7 \mathrm{~b}$ & $3,7 b$ & 4,5 & 4,4 \\
\hline 124g.ha- & 7 DAPC & $1,2 \mathrm{~b}$ & $1,7 \mathrm{c}$ & $2,3 \mathrm{~b}$ & $3,3 \mathrm{~b}$ & $3,4 \mathrm{~b}$ & 4,0 & 4,3 \\
\hline 90g.ha ${ }^{-1}$ & 7 DAPC & $1,0 \mathrm{~b}$ & $1,4 \mathrm{c}$ & $1,9 \mathrm{~b}$ & $2,9 \mathrm{~b}$ & $3,5 \mathrm{~b}$ & 4,8 & 4,4 \\
\hline
\end{tabular}

*Dias Antes do Ponto de Colheita

Médias seguidas da mesma letra, na coluna, não diferiram significativamente, a $5 \%$ de probabilidade, pelo teste de Duncan.

TABELA 8 - Efeito de concentrações e épocas de aplicação de $\mathrm{AVG}$, na porcentagem de cor vermelha dos frutos, na cv.Imperial Gala, em diversas datas de avaliações. Fraiburgo-SC, 2004.

\begin{tabular}{|c|c|c|c|c|c|c|c|c|}
\hline \multirow{2}{*}{\multicolumn{2}{|c|}{$\begin{array}{c}\text { Tratamentos } \\
\text { AVG }\end{array}$}} & \multicolumn{7}{|c|}{$\%$ de cor vermelha } \\
\hline & & $12-02$ & $19-02$ & $26-02$ & $04-03$ & $10-03$ & $17-03$ & $26-03$ \\
\hline $0 \mathrm{~g} \cdot \mathrm{ha}^{-1}$ & & $81,6 a$ & 80,4 n.s. & 92,8 n.s. & 92,4 n.s. & 89,6 n.s. & 92,4 n.s. & 94,0 n.s \\
\hline 124g.ha ${ }^{-1}$ & 30 DAPC* & $48,0 \mathrm{~b}$ & 63,2 & 85,2 & 86,4 & 89,6 & 93,2 & 95,6 \\
\hline $\begin{array}{l}\text { 62g.ha }{ }^{-1} \\
62 \mathrm{~g} \cdot \mathrm{ha}^{-1}\end{array}$ & $\begin{array}{l}30 \mathrm{DAPC}+ \\
15 \mathrm{DAPC}\end{array}$ & $53,2 b$ & 46,8 & 80,0 & 76,2 & 85,6 & 94,8 & 90,0 \\
\hline 90g.ha ${ }^{-1}$ & 15 DAPC & $54,0 \mathrm{~b}$ & 48,0 & 74,8 & 73,2 & 80,8 & 92,4 & 92,0 \\
\hline 124g.ha ${ }^{-1}$ & $7 \mathrm{DAPC}$ & $53,6 \mathrm{~b}$ & 49,2 & 74,0 & 77,2 & 73,2 & 89,2 & 91,6 \\
\hline 90g.ha ${ }^{-1}$ & 7DAPC & $54,0 \mathrm{~b}$ & 42,0 & 68,0 & 75,2 & 76,4 & 87,6 & 87,2 \\
\hline
\end{tabular}

* Dias Antes do Ponto de Colheita

Médias seguidas da mesma letra, na coluna, não diferiram significativamente, a $5 \%$ de probabilidade, pelo teste de Duncan. 
TABELA 9 - Efeito de concentrações e épocas de aplicação de AVG, no peso médio dos frutos em função da data de colheita (média por planta), na cv.Imperial Gala. Fraiburgo-SC, 2004.

\begin{tabular}{|c|c|c|c|c|c|c|c|c|}
\hline \multirow{2}{*}{$\begin{array}{c}\text { Tratamentos } \\
\text { AVG }\end{array}$} & \multicolumn{6}{|c|}{ Peso médio dos frutos - $g$} & \multirow{2}{*}{ Prod./ planta - kg } & \multirow{2}{*}{ Média geral } \\
\hline & $12-02$ & 19-02 & 23-02 & 02-03 & $10-03$ & 18-03 & & \\
\hline 0 g.ha $^{-1}$ & 112,8 & 118,4 & $113,5 \mathrm{c}$ & $102,5 \mathrm{~b}$ & - n.s. & - & $44,4 \mathrm{a}$ & $110,7 \mathrm{~b}$ \\
\hline 124g.ha" ${ }^{-1} 30$ DAPC* & - & - & $127,4 \mathrm{abc}$ & $127,6 \mathrm{a}$ & 121,0 & $104,5 \mathrm{~b}$ & $41,8 \mathrm{ab}$ & $121,2 \mathrm{a}$ \\
\hline $\begin{array}{ll}\text { 62g.ha } & 30 \text { DAPC + } \\
\text { 62g.ha } & 15 \text { DAPC }\end{array}$ & - & - & 145,9 a & $133,2 \mathrm{a}$ & 123,9 & $116,1 \mathrm{ab}$ & $51,3 \mathrm{a}$ & $125,5 \mathrm{a}$ \\
\hline 90g.ha' ${ }^{-1} \quad 15$ DAPC & - & - & $144,7 \mathrm{a}$ & $131,3 \mathrm{a}$ & 131,4 & $121,5 \mathrm{a}$ & $46,2 \mathrm{a}$ & $128,8 \mathrm{a}$ \\
\hline 124g.ha" 7 DAPC & - & - & $123,3 \mathrm{bc}$ & $129,7 \mathrm{a}$ & 120,0 & $113,4 \mathrm{ab}$ & $33,8 \mathrm{~b}$ & $121,4 \mathrm{a}$ \\
\hline 7 DAPC & - & - & $133,2 \mathrm{ab}$ & $121,8 \mathrm{a}$ & 124,3 & $111,1 \mathrm{ab}$ & $47,2 \mathrm{a}$ & $120,8 \mathrm{a}$ \\
\hline
\end{tabular}

*Dias Antes do Ponto de Colheita

Médias seguidas da mesma letra, na coluna, não diferiram significativamente, a $5 \%$ de probabilidade, pelo teste de Duncan.

TABELA 10 - Efeito de concentrações e épocas de aplicação de $\mathrm{AVG}$, na porcentagem de queda de frutos da cv.Imperial Gala, em função da data de colheita. Fraiburgo-SC, 2004.

\begin{tabular}{|c|c|c|c|c|c|c|}
\hline \multirow{2}{*}{$\begin{array}{c}\text { Tratamentos } \\
\text { AVG }\end{array}$} & \multicolumn{6}{|c|}{ \% de frutos caídos por data } \\
\hline & 23-02 & 26-02 & $04-03$ & $10-03$ & $17-03$ & $25-03$ \\
\hline 0 g.ha $^{-1}$ & 1,26 & 1,14 & $6,14 \mathrm{a}$ & $9,88 \mathrm{a}$ & $15,96 \mathrm{a}$ & $24,2 \mathrm{a}$ \\
\hline 124 g.ha $^{-1} 30$ DAPC $*$ & 0,24 & 0,20 & $1,46 \mathrm{~b}$ & $5,16 \mathrm{~b}$ & $16,40 \mathrm{a}$ & $25,0 \mathrm{a}$ \\
\hline $\begin{array}{ll}62 \mathrm{~g}_{\mathrm{ha}}{ }^{-1} & 30 \mathrm{DAPC}+ \\
62 \mathrm{~g}^{-1} \mathrm{ha}^{-1} & 15 \mathrm{DAPC}\end{array}$ & 0,30 & 0,00 & $0,48 \mathrm{~b}$ & $2,06 \mathrm{~b}$ & $5,54 \mathrm{~b}$ & $24,3 \mathrm{a}$ \\
\hline 90 g.ha $^{-1} \quad 15$ DAPC & 0,14 & 0,08 & $0,64 \mathrm{~b}$ & $2,76 \mathrm{~b}$ & $5,94 \mathrm{~b}$ & $20,8 \mathrm{a}$ \\
\hline 124 g.ha $^{-1} 7$ DAPC & 0,28 & 0,06 & $0,50 \mathrm{~b}$ & $2,34 \mathrm{~b}$ & $6,18 b$ & $9,3 b$ \\
\hline 90 g.ha ${ }^{-1}$ & 0,12 & 0,06 & $0,88 \mathrm{~b}$ & $2,66 \mathrm{~b}$ & $9,68 \mathrm{~b}$ & $19,5 \mathrm{a}$ \\
\hline
\end{tabular}

* Dias Antes do Ponto de Colheita

Médias seguidas da mesma letra, na coluna, não diferiram significativamente, a $5 \%$ de probabilidade, pelo teste de Duncan.

TABELA 11 - Efeito de época de aplicação de AVG, na queda de frutos por planta e frutos com rachadura peduncular. $\mathrm{Cv}$ Imperial Gala. Fraiburgo-SC, 2003.

\begin{tabular}{lccc}
\hline $\begin{array}{c}\text { Época da aplicação } \\
\text { AVG }\end{array}$ & $\begin{array}{c}\text { Número de frutos } \\
\text { caídos/planta }\end{array}$ & $\begin{array}{c}\text { \% de frutos caídos } \\
\text { \% de frutos com rachadura } \\
\text { peduncular -25/03 }\end{array}$ \\
\hline 45 DAPC* & $11,8 \mathrm{a}$ & 11,2 & 36 \\
30 DAPC & $9,5 \mathrm{ab}$ & 9,4 & 10 \\
15 DAPC & $5,9 \mathrm{c}$ & 6,8 & 18 \\
7 DAPC & $8,1 \mathrm{bc}$ & 8,6 & 22
\end{tabular}

*Dias Antes do Ponto de Colheita

Médias seguidas da mesma letra, na coluna, não diferiram significativamente, a $5 \%$ de probabilidade, pelo teste de Duncan.

\section{CONCLUSÕES}

1-O efeito do AVG no retardamento da maturação da cv. Gala é dependente da concentração e da época de aplicação. Aplicações de AVG mais próximas do ponto de colheita comercial, 7 DAPC, foram mais efetivas no controle de queda de frutos précolheita e no retardamento da perda de firmeza do que aplicações 30 ou 45 DAPC.

2-Doses de 124 e 90 g.ha-1 de AVG são igualmente efetivas quando aplicadas 7 ou 15 dias antes do ponto de colheita comercial, dependendo do percentual de retardamento desejado.

\section{REFERÊNCIAS}

AMARANTE, C.V.T.; SIMIONI, A.; MEGGUER, C.A.; BLUM, L.E.B. Effect of aminoethoxyvinilglicine (AVG) on preharvest fruit drop and maturity of apples. Revista Brasileira Fruticultura, Jaboticabal,, v.24, n.3, p. 661-664, 2002.

ARGENTA, L.C. Concentração interna de etileno e maturação de maçãs 'Gala', 'Golden Delicious' e 'Fuji'. Revista Brasileira de Fruticcultura, Jaboticabal, v.15, n. ,p.125-132, 1992.

AUTIO, W.R.; BRANLAGE, W. Effect of AVG on maturation, ripening and storage of apples. Journal of the American Society Horticultural Science, Alexandria, v.107, p.974-1077, 1982.

BRAMLAGE, W.J.; GREENE, D.W.; AUTIO, W.R.; MCLAUGHLIU, J.M. Effects of Aminoethoxyvinylglicine on internal Ethylene concentrations and storage of apples. Journal of the American Society Horticultural Science, Alexandria, v.105, n.6, p.847-851, 1980.

BYERS, R.E. Effects of aminoethoxyvinylglicine (AVG) on preharvest fruit drop, maturity and cracking of several apple cultivars. Journal of Tree Fruit Production, Chicago, v. 2, p.77-97, 1997.

CHUN, J.; PARK, M.; HWANG, Y.; LEE, J. Effect of AVG on harvest drop and fruit quality in tsugaru apples. Journal of the 
Korean Society for Horticultural Science, Suwon, v.38, p.147-152, 1997.

GREENE, D. W. Development of aminoethoxyvinylglicine (AVG) to retard preharvest in apples. Acta Horticulturae, Wageningen, n.527, p.105-109, 2000.

GREENE, D.W. Endogenous hormones and bioregulators use on apple. In: FERRE, D.C.; WARRINGTON, J.J. Apples, botany, productions use. Wallinford: Cabi Publishing, 2003. p.660.

GREENE, D.W. Ethylene-based preharvest growth regulators. In: MAIB, K., ANDREWS, P., LANG, G.; MULLINEX, K. Tree Fruit Physiology: growth and development. Washington: Good Fruit Grower, 1996. p.149-159.

GREENE, D.W. Preharvest drop control and maturity of 'Delicious' apples as affected by aminoethoxyvinylglicine (AVG). Journal of Tree Fruit Production, Chicago, ,v.3, p.1-10, 2002.

LURIE, S. Manipulation fruit development and storage quality using growth regulators. In: PLANT growth regulators in agriculture and horticulture. London: Food Products Prens, 2000.p.196

SCHUPP, J.R.; GREENE, D.W. Effect of Aminoethoxyvinylglicine(AVG) on preharvest drop, fruit quality, and maturation of 'McIntosh' apples. I. Concentration and timing of dilute applications of AVG. HortScience, Alexandria, v. 39,n. 5, p.1030-1035, 2004.

SMOCK, R.M.; GROSS, C.R. Some effect of some hormone materials on the respiration and softening rates of apples. Proceedings of the American Society Horticultural Science, Alexandria, v. 49, p.67-77, 1947.

STOVER, E.;FARGIONI, M.J.; WATKINS, C.B.; IUNGERMAN, K.A. Harvest management of Marsall 'MacIntosh' Apples: Effect of AVG, ANA, Ethephon and Summer pruning on preharvest drop and fruit quality. HortScience, Alexandria, v.38, n.6, p.1093-1099, 2003. 